\title{
Pathogenicity of Beauveria bassiana against the tiger moth Atteva sciodoxa (Lepidoptera: Yponomeutidae)
}

\begin{abstract}
Seven isolates of Beauveria bassiona were screened for pathogenicity and infectivity at a concentration of $5 \times 107$ conidia mL-1 against Attevo sciodoxa at $27 \hat{\mathrm{A}} \pm 2 \hat{\mathrm{A}}^{\circ} \mathrm{C}$ and $75 \hat{\mathrm{A}} \pm 5 \%$ relative humidity with $12 \mathrm{~h}$ photoperiod. Based on screening results, isolates Bba-Pp and FS11 were further bioassayed at $1 \times 106,5 \times 106$ and 1x107 conidia mL-1. All the isolates were found to be pathogenic. However, the infectivity varied significantly among the isolates. The earliest mortality was recorded three days after inoculation, The most virulent isolate, BbaPp, caused $100 \%$ mortality with a median infective time (ET50) of 3.6 days on day seven following inoculation while FS-11 caused 83,3\% mortality with an ET50 value of 4.1 days, Bba-S13 was the least infective isolate with $24.9 \%$ mortality and 15.3 days of median effective time. Mycelia appeared on 24 to $48 \mathrm{~h}$ old cadavers, The highest level of sporulation on two-week old cadavers was $150.6 \times 105$ Bba-Pp conidia mg -1 cadaver while the lowest was 12.23x105 Bba-S13 conidia. The median effective concentration (EC50) of Bba-Pp was 9.89x105 conidia mL-1 while that of FS-11 was $3.85 \times 106$ conidia mL-1. The ET50 values 1x106 1x107 conidia mL-1 of Bba-Pp ranged between 7.0 and 4.4 days, respectively, while that of FS-11 were 10.3 and 5.8 days. A strong negative correlation was found between inoculum concentrations and food consumption $(\mathrm{R} 2=-0.99)$. The infection by Bba-Pp and FS-11I resulted in 55.8 to $72.5 \%$ reduction in food consumption by A. sciodoxa compared to the controls.
\end{abstract}

Keyword: Beuveria bassiana; Pathogenicity; Atteva sciodoxa; Tiger moth; Eurycoma longifolia; Biological control 